\title{
Abschätzung der Standsicherheit von annähernd unendlich langen Kriechhängen
}

\author{
Wolfgang Fellin *
}

17. Oktober 2019

This is the peer-reviewed version of the following article: Fellin, W. (2011): Abschätzung der Standsicherheit von annähernd unendlich langen Kriechhängen. geotechnik 34(1):22-31, which has been published in final form at https://doi.org/10.1002/gete.201000018. This article may be used for non-commercial purposes in accordance with Wiley Terms and Conditions for Use of SelfArchived Versions.

Langsam kriechende Hänge stellen eine eventuelle Bedrohung für darunter liegende Siedlungen und Infrastrukturen dar. Bereits in den 30er Jahren wurde eine Lösung zur Ermittlung des Spannungszustandes in einer kriechenden Schicht konstanter Dicke auf einer unendlich langen Böschung entwickelt, die als Grundlage für die Untersuchung von Kriechhängen dienen kann. In diesem Beitrag wird diese Lösung erweitert und gemeinsam mit dem Grenzzustand nach Matsuoka-Nakai verwendet, um die Sicherheit von Kriechhängen abschätzen zu können, welche annähernd als unendliche Böschung modellierbar sind.

Slowly creeping slopes are a potential threat to downhill settlements and infrastructure. The stress state in a creeping layer with constant thickness on an infinite slope can be calculated based on a solution from the thirties of the past century. This solution is extended and combined with the Matsuoka-Nakai limit state criterion. The safety of a roughly infinite creeping slope can be estimated with the presented approach.

\section{Einleitung}

Langsam kriechende Hänge stellen eine eventuelle Bedrohung für darunter liegende Infrastrukturen dar, wenn sie aus dem quasistationären Kriechen in eine beschleunigte Bewegung übergehen. Die Stabilität solcher Kriechhänge sollte daher einigermaßen verlässlich abgeschätzt werden können.

\footnotetext{
*Arbeitsbereich für Geotechnik und Tunnelbau, Institut für Infrastruktur, Universität Innsbruck, e-mail: wolfgang.fellin@uibk.ac.at .
} 


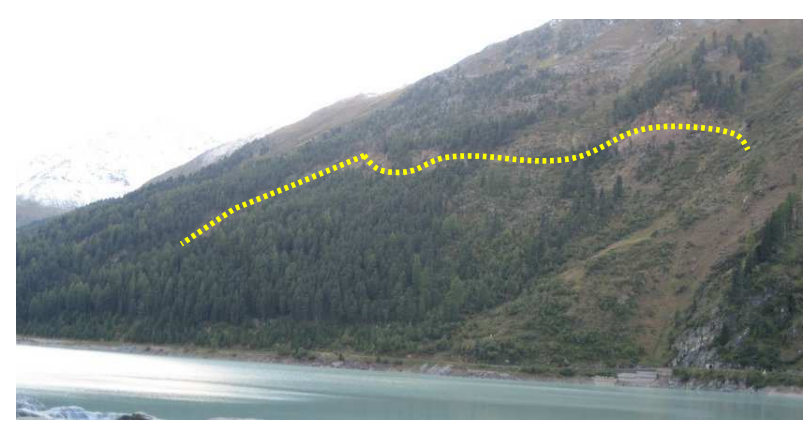

Abbildung 1: Derzeit kriechende unterste Scholle des Hochmais-Atemkopf Hanges, Kaunertal. Die gestrichelte Linie bezeichnet ungefähr den Anriss von 1964.

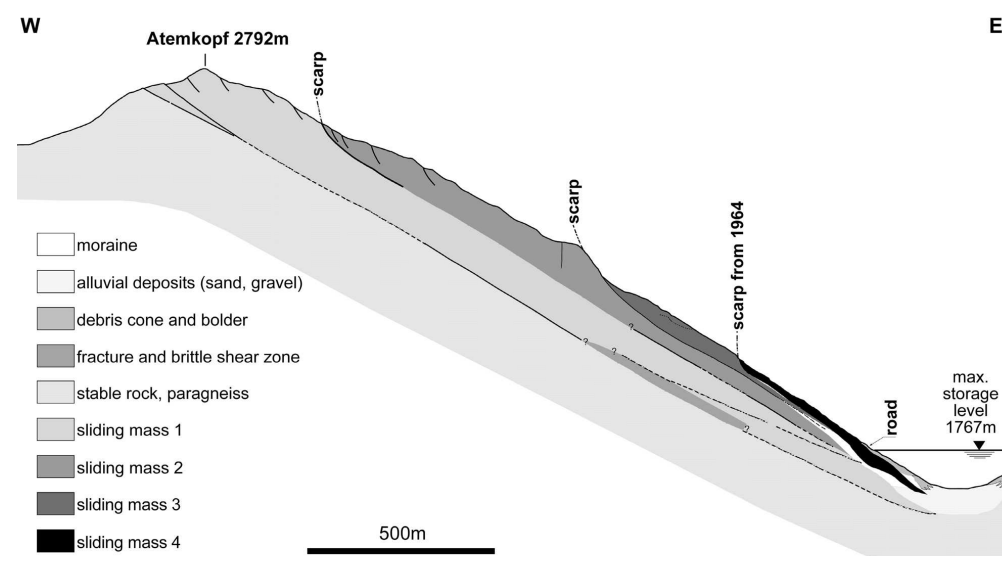

Abbildung 2: Geologisches Profil Hochmais-Atemkopf, Kaunertal: derzeit kriecht die unterste Scholle (slinding mass 4) [aus 26] 
Es kann entweder ein ganze Schicht kriechen oder lediglich eine tiefer liegende Zone, welche die darüber liegenden Massen mit transportiert. Die kriechenden Schichten können z.B. aus Permafrostböden oder zerriebenen Gesteinszonen (Kakiriten) bestehen. Im Fall des Kriechhanges HochmaisAtemkopf im Kaunertal (Abb. 1 und 2) ist die Kriechzone der untersten Scholle eine überschobene Gletschermoräne [2]. Dieser Hang wird seit mehr als 40 Jahren sorgfältig beobachtet (u.a. durch Drahtextensiometer in einem Kontrollstollen, Abb. 3) und weist leicht schwankende Kriechraten von 3 bis $4 \mathrm{~cm}$ pro Jahr auf [26].

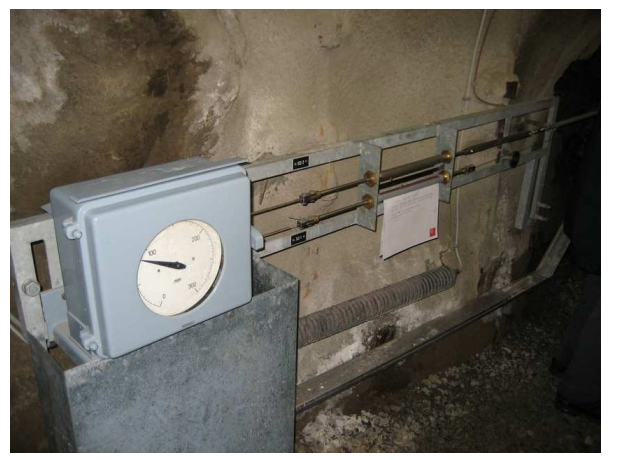

Abbildung 3: Messtation des Drahtextensiometers im Kontrollstollen der Kriechscholle HochmaisAtemkopf.

Langsame Massenbewegungen und kriechende Schneeschichten haben viel gemeinsam. Haefeli [9] entwickelte eine kinematische Lösung zur Berechnung des Spannungszustandes in einer geneigten Schneeschicht. Diese Lösung wurde bereits von Körner [13] zur Stabilitätsuntersuchung von Kriechhängen vorgeschlagen. Bei bekannter Geschwindigkeitsverteilung im Hang, und mit einigen wenigen plausiblen Annahmen, können die Hauptspannungen in einer kriechenden Schicht nach Haefeli aus Messungen der Oberflächengeschwindigkeit ermittelt und anschließend in Hinblick auf den Grenzzustand untersucht werden. Die Stärke der kinematischen Lösung ist, dass sie lediglich das Zusammenfallen der Hauptrichtungen der Verzerrungsrate und der Spannung voraussetzt, aber ansonsten kein spezielles Materialmodell zugrunde legt. Haefeli [9] hat seine Lösung graphisch gefunden und lediglich Aussagen über die Spannungen in einer Schnittebene längs des Hanges getroffen.

Im Folgenden wird eine algebraisch abgeleitete auf den vollständigen Spannungstensor erweiterte kinematische Lösung verwendet, um für annähernd unendlich lange Kriechhänge eine erste Abschätzung der Stabilität zu erhalten.

\section{Spannungszustand in einer Kriechschicht}

Zur Berechnung der Eigengewichtsspannungen in einer kriechenden Schicht konstanter Dicke auf einer unendlich langen Böschung gehen wir zunächst wie bei der Ermittlung des Rankine'schen Spannungszustandes in einem unendlich langen Hang vor [vgl. z.B. 12]. Dazu betrachten wir eine vertikale Lamelle der Breite $b \cos \beta$ und der Tiefe $t$ einer Schicht mit konstanter Dicke auf einem um $\beta$ geneigten Hang, Abb. 4. Da der Hang in der $x_{3}$-Richtung unendlich ist, gilt ein ebener Verzerrungszustand.

An der herausgeschnittenen Lamelle (Abb. 6) wirken zwei auf den Seiten angreifenden Kräfte $S_{1}$ und $S_{2}$. In einer unendlich langen Böschung sind alle Nachbarlamellen gleichberechtigt und damit 


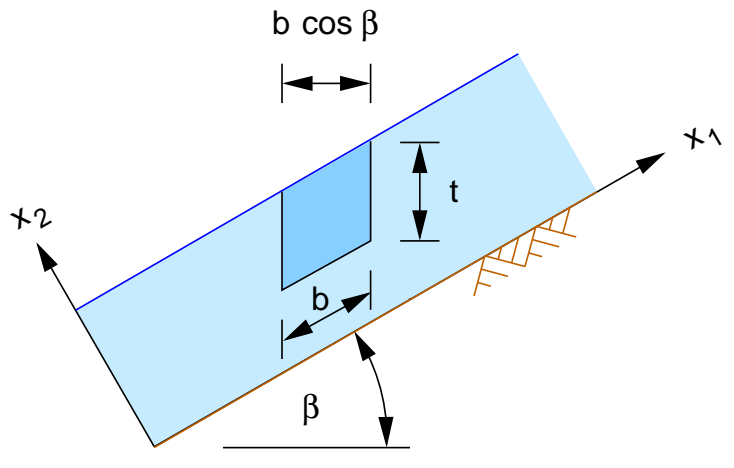

Abbildung 4: Lamelle in einer unendlich langen Böschung: die Spannungen im Schnitt in der Tiefe $t$ sind gesucht.

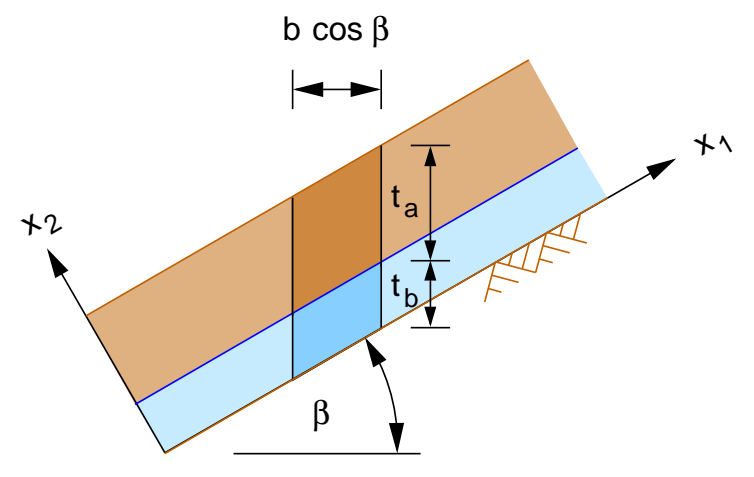

Abbildung 5: Situation mit begrenzter Kriechzone.

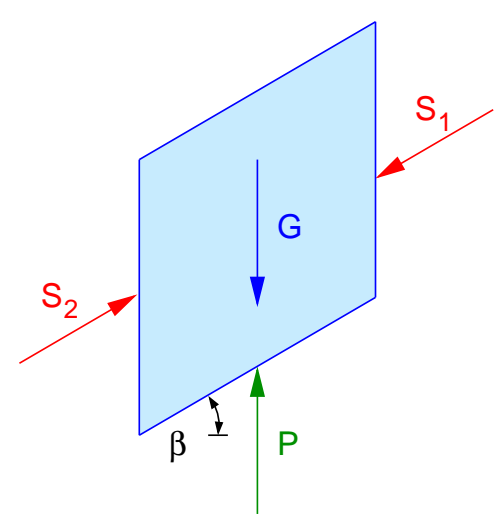

Abbildung 6: Kräfte an der Lamelle. 
ist $S_{1} \equiv-S_{2}$. Das Eigengewicht $G$ wird deshalb direkt von der senkrechten haltenden Kraft $P$ aufgenommen.

Der Vertikaldruck an der Sohle der Lamelle (Abb. 4) ist

$$
p_{v}=\frac{G}{b}=\frac{\gamma t b \cos \beta}{b}=\gamma t \cos \beta
$$

Für die Situation einer begrenzten Kriechzone (Abb. 50) ist der Vertikaldruck an der Sohle der Kriechzone

$$
p_{v}=\frac{G_{a}+G_{b}}{b}=\frac{\left(\gamma_{a} t_{a}+\gamma_{b} t_{b}\right) b \cos \beta}{b}=\left(\gamma_{a} t_{a}+\gamma_{b} t_{b}\right) \cos \beta .
$$

Dieser Druck kann in die Normalspannung $\sigma_{22}$ und die Schubspannung $\sigma_{21}$ aufgeteilt werden

$$
\begin{aligned}
\sigma_{22} & =p_{v} \cos \beta \\
\sigma_{21} & =p_{v} \sin \beta .
\end{aligned}
$$

Hier und im Folgenden sind effektive Spannungen gemeint.

Damit ist der Spannungszustand wegen $\sigma_{12}=\sigma_{21}$ fast bekannt

$$
\boldsymbol{\sigma}=\left(\begin{array}{ll}
\sigma_{11} & \sigma_{12} \\
\sigma_{21} & \sigma_{22}
\end{array}\right)=\left(\begin{array}{ll}
\sigma_{11}=? & p_{v} \sin \beta \\
p_{v} \sin \beta & p_{v} \cos \beta
\end{array}\right)
$$

Es fehlt lediglich die Spannung $\sigma_{11}$ aus den Lamellenzwischenkräften $S$. Zur Ermittlung dieser fehlenden Spannung hat Haefeli [9] eine kinematische Lösung vorgeschlagen.

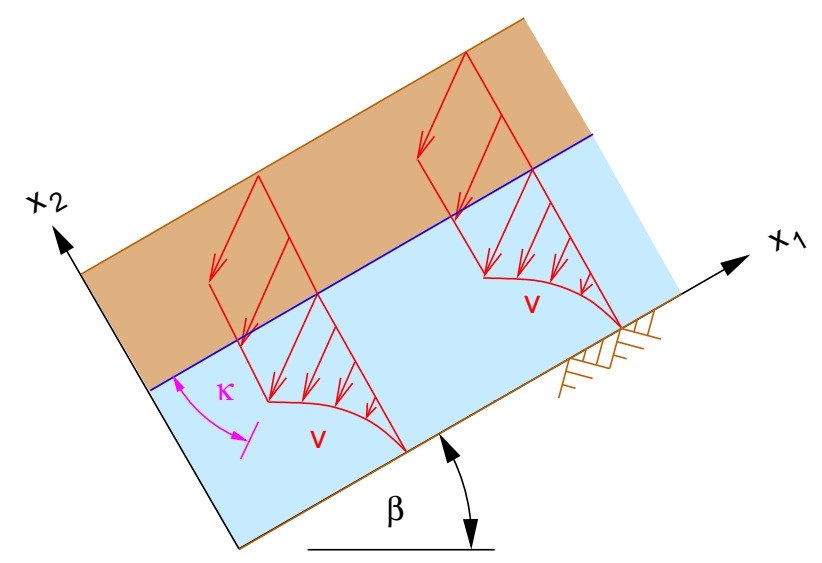

Abbildung 7: Geschwindigkeitsfeld in einer unendlich langen Kriechschicht mit darüber liegender nicht kriechender Schicht.

Für einen bekannten Kriechwinkel $\kappa$ (Abb. 7) und unter Annahme paralleler Geschwindigkeitsvektoren sowie der Koaxialität von Spannung und Verzerrungsrate lassen sich analog zu Haefeli die Hauptspannungen $\sigma_{I} \geq \sigma_{I I} \geq \sigma_{I I I}$ in der Kriechschicht ermitteln (siehe Anhang @ي): 


$$
\begin{aligned}
\sigma_{I} & =p_{v} \frac{\cos (\beta+\kappa)+\sin (\beta)}{\cos \kappa} \\
\sigma_{I I} & =p_{v} \frac{\cos (\beta+\kappa)-\sin (\beta) \sin (\kappa)}{\cos \kappa} \\
\sigma_{I I I} & =p_{v} \frac{\cos (\beta+\kappa)-\sin (\beta)}{\cos \kappa}
\end{aligned}
$$

\section{Materialverhalten in der Kriechschicht}

Kriechende Böden zeigen einen ausgeprägt nicht linearen Zusammenhang zwischen Kriechrate und Spannungsdeviator [z.B.: 6, 19, 25, 28, 32, 33]. Qualitativ verhält sich kriechender Boden im lastgesteuerten Triaxialversuch wie in Abb. 8. Ob die angedeutete Grenze für die aufnehmbare Deviatorspannung $\left(\sigma_{1}-\sigma_{3}\right)_{\max }$ (im Sinne einer Asymptote an den Kriechvorgang) existiert, und wie groß diese im Vergleich zur maximalen Deviatorspannung im weggesteuerten Triaxialversuch $\left(\sigma_{1}-\sigma_{3}\right)_{f}$ ausfällt, ist noch unklar.

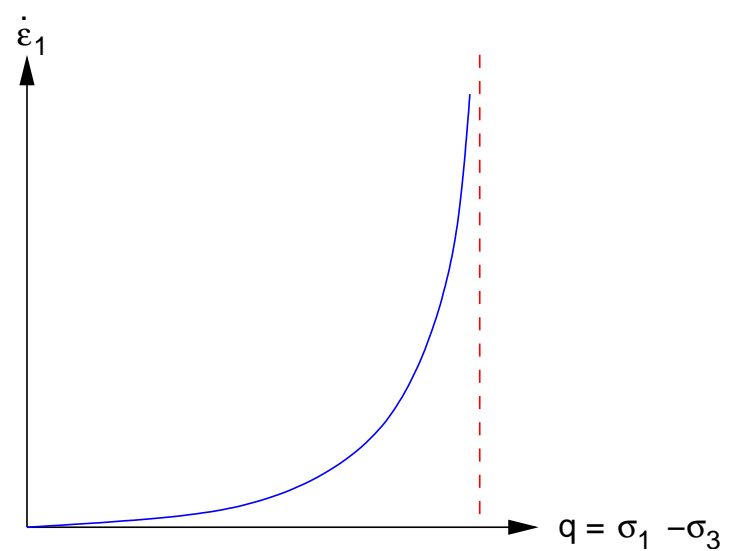

Abbildung 8: Qualitative Abhängigkeit der vertikalen Stauchungsrate $\dot{\varepsilon}_{1}$ im lastgesteuerten Triaxialversuch mit konstanter Vertikalspannung $\sigma_{1}>\sigma_{2}=\sigma_{3}$. Die vertikale gestrichelte Linie bezeichnet die maximal aufnehmbare Deviatorspannung $\left(\sigma_{1}-\sigma_{3}\right)_{\max }$

Es gibt eine große Anzahl von Materialmodellen für kriechende Böden, siehe z.B. die umfangreiche Zusammenstellung von Vulliet und Hutter [32]. Für die Fragestellung der Standsicherheit ist weniger die genaue Modellierung des Zusammenhanges zwischen Kriechrate und Spannungen interessant, als mehr die Modellierung eines Kriechbruches. Deshalb sind Materialmodelle interessant, welche ein Versagenskriterium des Bodens berücksichtigen. Vulliet und Hutter [32] modellieren das Kriechverhalten von Böden mit

$$
\dot{\varepsilon}=\frac{2}{\sqrt{3}} A\left[\frac{\sigma_{1}-\sigma_{3}}{\left(\sigma_{1}-\sigma_{3}\right)_{f}}\right]^{m},
$$

worin $A$ und $m$ Materialkonstanten sind, sowie $\left(\sigma_{1}-\sigma_{3}\right)_{f}$ die über ein Versagenskriterium nach Drucker-Pragen 1 postulierte Festigkeit, und geben eine gute Übereinstimmung mit Experimenten an

\footnotetext{
${ }^{1}$ Eine Adaptierung des Kriechmodelles von Vulliet und Hutter [32] auf ein Versagenskriterium nach Mohr-Coulomb wird derzeit von Schneider-Muntau [23] erarbeitet.
} 
Ton bis $0,8 \cdot\left(\sigma_{1}-\sigma_{3}\right)_{f}$ an. Dieses Kriechmodell verknüpft jeden Spannungszustand mit einer Stauchungsrate. Insbesondere liefert eine Extrapolation des Modelles (9) für Spannungszustände jenseits des Versagenskriteriums, $\left(\sigma_{1}-\sigma_{3}\right)>\left(\sigma_{1}-\sigma_{3}\right)_{f}$, eine zwar relativ große aber endliche Stauchungsrate. Damit ist kein Versagen im Sinn einer maximal aufnehmbaren Deviatorspannung $\left(\sigma_{1}-\sigma_{3}\right)_{\max }$ wie in Abb. 8 modellierbar.

Inwieweit sich der Grenzzustand von last- und weggesteuerten Versuchen vergleichen lässt, ist noch nicht gänzlich geklärt. Oft wird deshalb die maximale Deviatorspannung für beide Versuchsarten gleichgesetzt. Allerdings finden di Prisco und Imposimato [4], dass das Versagen von locker gelagerten Sanden in lastgesteuerten Triaxialversuchen deutlich früher als bei weggesteuerten auftritt.

Andererseits zeigen lastgesteuerte drainierte Triaxialversuche an vier dicht gelagerten Proben aus Kakirit und fünf dicht gelagerten Proben aus Moränenmaterial [20, 21] ein Versagen der Probe bei Spannungen $\left(\sigma_{1}-\sigma_{3}\right)_{\max } \approx(0,9 \ldots 1,2) \cdot\left(\sigma_{1}-\sigma_{3}\right)_{f}$ des Versagenszustandes von weggesteuerten Versuchen am gleichen Material. Die weggesteuerten Versuche wurden wie üblich mit Belastungsgeschwindigkeiten passen zu den Konsolidierungszeiten gefahren. Dies ergab Stauchungsraten zwischen $10^{-6}$ und $10^{-5} 1 / \mathrm{s}$. Im Mittel trat Versagen bei $\left(\sigma_{1}-\sigma_{3}\right)_{\max }=1,05 \cdot\left(\sigma_{1}-\sigma_{3}\right)_{f}$ ein. Die Belastung wurde in den Versuchen sprunghaft in Stufen erhöht. In Laststufen ohne Versagen ging die anfänglich hohe Kriechrate der transienten Kriechphase in die niedrigere und konstante Kriechrate des stationäres Kriechen (ca. $10^{-6} 1 / \mathrm{s}$ ) über, Abb. 9. In Laststufen mit Versagen trat nach kurzem stationären Kriechen beschleunigtes (tertiäres) Kriechen auf 2 oder es gab gar kein Abklingen der Anfangsrate auf den stationären Zustand. Versagenszustände waren in den Kriechversuchen von Dilatanz begleitet, wogegen das Volumen der Proben bei stationärem Kriechen ungefähr konstant blieb. Es wird hier vermutet, dass tertiäres Kriechen nur bei dilatantem Verhalten auftritt, da die mit der Dilatanz verknüpfte Auflockerung zu einem graduellen Festigkeitsverlust führt. Der Übergang von volumenkonstantem zu dilatantem Kriechen liegt für dichte Böden laut den vorliegenden Untersuchungen in der Nähe der maximalen Deviatorspannung aus zugehörigen weggesteuerten Versuchen. Ein gewisse Unsicherheit stellt die Tatsache dar, dass aufgrund der langen Versuchsdauern nicht verlässlich geprüft werden kann, ob eventuell auch in niedrigeren Laststufen tertiäres Kriechen auftritt, wenn nur lange genug gewartet wird.

Für diese Frage sind die Untersuchungen von Krieg [14] an bindigen Böden in triaxialen Kriechversuchen interessant. Er vermutet aus seinen Ergebnissen, dass es keinen Kriechbruch für drainierte Verhältnisse gibt, solange der mobilisierte Reibungswinkel $\varphi_{m}$ kleiner als der kritische Reibungswinkel $\varphi_{c}$ des Materials ist. Der mobilisierte Reibungswinkel errechnet sich aus den effektiven Spannungen $\varphi_{m}=\left(\sigma_{1}-\sigma_{3}\right) /\left(\sigma_{1}-\sigma_{3}\right)$, der kritische Reibungswinkel gilt für volumenkonstantes Scheren. Zudem zeigt er, dass der kritische Reibungswinkel für sein Proben praktisch nicht der Belastungsgeschwindigkeit abhängt. Leroueil und Marques [15] fassen aus zusammengestellten Versuchen zusammen: Normalkonsilidierte Tone versagen im Kriechversuch bei der kritischen Scherfestigkeit, und die kritische Scherfestigkeit hängt nicht von der Belastungsgeschwindigkeit ab.

Für die Abschätzung der Standsicherheit sollte deshalb für dichte Böden in erster Näherung die kritische Scherfestigkeit aus weggesteuerten Standardversuchen als Begrenzung für verträgliche Kriechspannungen verwendet, d.h. für triaxiale Bedingungen: $\left(\sigma_{1}-\sigma_{3}\right)_{\max }=\left(\sigma_{1}-\sigma_{3}\right)_{f, c}$. Für locker gelagerte

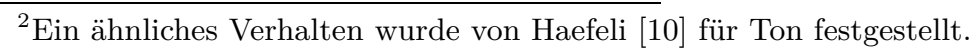



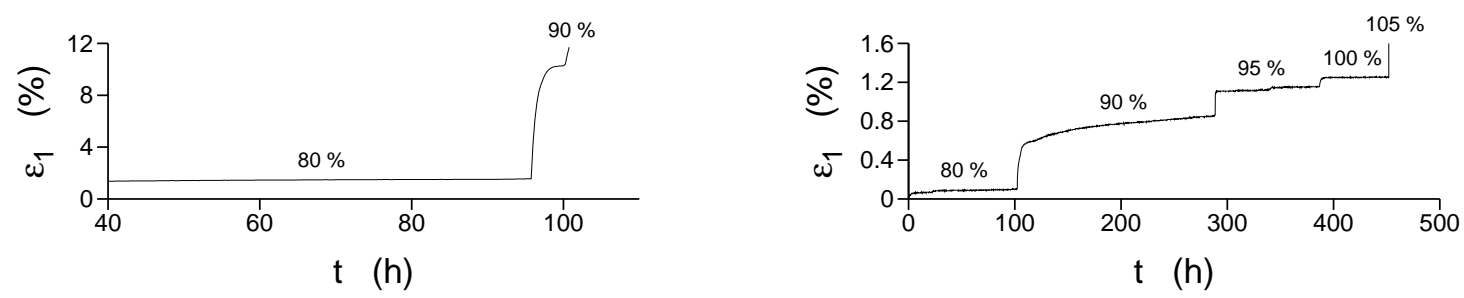

Abbildung 9: Zunahme der Axialstauchung $\varepsilon_{1}$ über die Zeit $t$ in Kriechversuchen an Kakirit: die Prozentzahlen bezeichnen die Größe der Deviatorspannung in Prozent der maximalen Deviatorspannung im weggesteuerten Versuch am gleichen Material; (links) Versagen bei Erhöhung der Deviatorspannung auf 90\% nach anfänglichem Übergang in stationäres Kriechen, (rechts) schlagartiges Versagen bei Erhöhung der Deviatorspannung auf $105 \%$ [21].

Böden sollten eventuell Abminderungen eingeführt werden. Typische Werte für die Scherparameter von Kriechzonenmaterialien wurden von Engl et al. [5] zusammengestellt.

Oft werden Rahmenscherversuche zur Bestimmung der Scherparameter verwendet. Diese sind aber schwierig zu interpretieren. Die im Rahmenscherversuch ermittelten Scherfestigkeiten hängen stark von der Bauart des Gerätes ab [8, 16] und können größer oder kleiner als jene im Triaxialversuch sein [1], siehe auch Anhang B, Aus solchen Versuchen abgeleitete Kennwerte sind deshalb mit besonderer Vorsicht zu verwenden.

\section{Abschätzung der Sicherheit}

Die Sicherheit einer unendlich langen Böschung kann durch den Vergleich der erforderlichen (mobilisierten) Scherfestigkeit mit der vorhanden definiert werden

$$
\eta:=\frac{\tau_{f, \mathrm{vor}}}{\tau_{f, \mathrm{erf}}}
$$

Werden beide Scherparameter $\varphi$ und $c$ gleich gewichtet, folgt

$$
\eta:=\frac{\tan \varphi_{\mathrm{vor}}}{\tan \varphi_{\mathrm{erf}}}=\frac{c_{\mathrm{vor}}}{c_{\mathrm{erf}}} .
$$

Der Kehrwert dieser Sicherheit wird als Ausnutzungsgrad bezeichnet.

\subsection{Unendlich lange Böschung in kohäsionslosem Boden}

In einer nicht kriechenden unendlich langen Böschung aus kohäsionslosem Material wird der erforderliche Reibungswinkel für das Grenzgleichgewicht üblicherweise aus der Scherfestigkeit $\tau_{f}=\sigma_{n} \tan \varphi$ berechnet. Hier ist die Normalspannung $\sigma_{n}=\sigma_{22}=p_{v} \cos \beta$ und aus $\tau_{f \text {,erf }}=\sigma_{21}=p_{v} \sin \beta$ folgt der erforderliche Reibungswinkel für Grenzgleichgewicht

$$
\varphi_{\text {erf }}=\beta \text {. }
$$




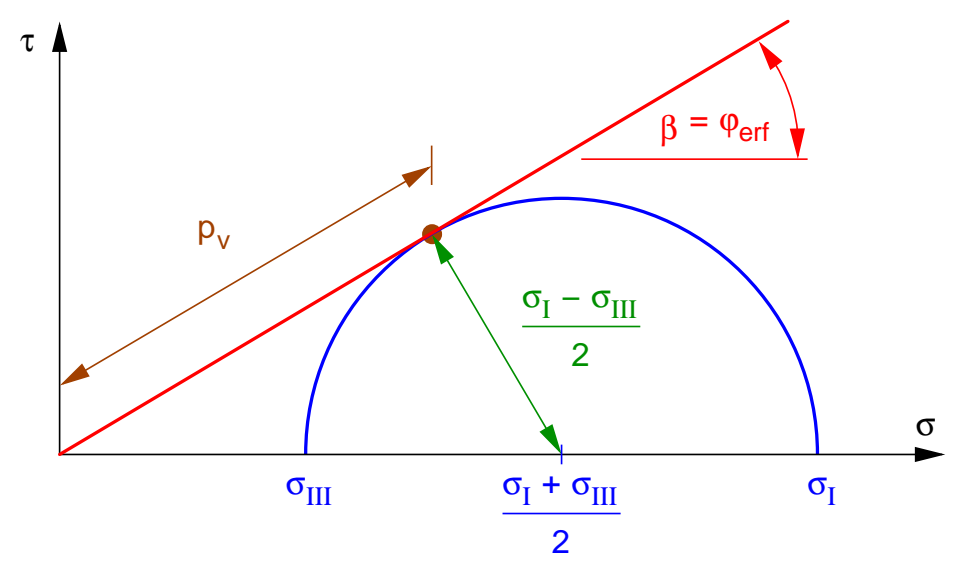

Abbildung 10: Spannungszustand in einer kohäsionslosen unendlich langen Böschung im Grenzgleichgewicht $\varphi_{\text {erf }}=\beta$.

Dies impliziert allerdings einen speziellen Spannungszustand, siehe Abb. 10. Aus $\left(\sigma_{I}-\sigma_{I I I}\right) / 2=$ $p_{v} \tan \beta$ und $\left(\sigma_{I}+\sigma_{I I I}\right) / 2=p_{v} / \cos \beta$ folgt

$$
\begin{aligned}
\sigma_{I} & =p_{v} \frac{1+\sin \beta}{\cos \beta} \\
\sigma_{I I I} & =p_{v} \frac{1-\sin \beta}{\cos \beta} .
\end{aligned}
$$

Aus einem Vergleich mit (6) und (8) ist leicht ersichtlich, dass dieser Spannungszustand in einer Kriechschicht nur für den Spezialfall $\kappa=-\beta$ auftritt, also für relativ stark dilatantes Kriechen.

\subsection{Grenzzustände im Hauptspannungsraum}

Mohr-Coulomb: Für eine Kriechschicht schlägt Körner [13] vor, den mittels der kinematischen Lösung ermittelten Spannungszustand (6,8) mit einem Versagenskriterium nach Mohr-Coulomb zu vergleichen

$$
\sigma_{I}-\sigma_{I I I} \leq\left(\sigma_{I}+\sigma_{I I I}\right) \sin \varphi+2 c \cos \varphi
$$

um zwischen stabilem und instabilem Kriechen zu unterscheiden. Für ein kohäsionsloses Material ergibt die Grenzbedingung (15) mit den Hauptspannungen (61) und (8)

$$
\sin \varphi_{\mathrm{erf}}=\frac{\sin \beta}{\cos (\beta+\kappa)} .
$$

Böden zeigen bei ebener Verformung (behinderte Querdehnung in Hanglängsrichtung) allerdings höhere Festigkeiten als im triaxialen Versuch. Dies wird im Versagenskriterium nach Mohr-Coulomb nicht berücksichtigt, Abb. 11. In der Kriechschicht ist die Querdehnung in der Tat behindert, weshalb die so ermittelten erforderlichen Scherparameter zu hoch ausfallen. 


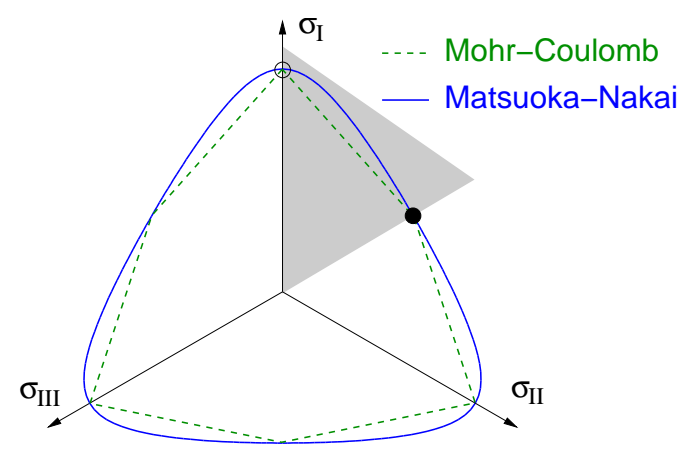

Abbildung 11: Versagenskriterium nach Mohr-Coulomb und Matsuoka-Nakai in einer Deviatorebene des Hauptspannungsraumes $\left(\varphi=35^{\circ}\right)$ : $\circ$ ist ein triaxialer Kompressionszustand mit $\sigma_{I}>\sigma_{I I}=\sigma_{I I I}, \bullet$ ist ein triaxialer Extensionszustand mit $\sigma_{I}=\sigma_{I I}>\sigma_{I I I}$. Der grau hinterlegte Bereich dazwischen bezeichnet Spannungszustände $\sigma_{I}>\sigma_{I I}>\sigma_{I I I}$, welche bei ebener Verformung auftreten.

Matsuoka-Nakai: Grenzzustände bei ebener Verformung können mit der Grenzbedingung nach Matsuoka und Nakai [17] realistischer erfasst werden (Abb. 11)

$$
\frac{I_{1} I_{2}}{I_{3}}=K_{\mathrm{MN}}=\frac{9-\sin ^{2} \varphi}{1-\sin ^{2} \varphi}
$$

mit den drei Invarianten des Spannungstensors $I_{1}, I_{2}$ und $I_{3}$. Mit den Hauptspannungen lautet (17)

$$
\frac{\left(\sigma_{I}+\sigma_{I I}+\sigma_{I I I}\right)\left(\sigma_{I} \sigma_{I I}+\sigma_{I I} \sigma_{I I I}+\sigma_{I I I} \sigma_{I}\right)}{\sigma_{I} \sigma_{I I} \sigma_{I I I}}=K_{\mathrm{MN}} .
$$

Kohäsion kann über eine Transformation der Normalspannung berücksichtigt werden: $\hat{\sigma}=\sigma+c / \tan \varphi$ [erweitertes Matsuoka-Nakai Kriterium: 18].

Für ein kohäsionsloses Material liefert Einsetzen des Spannungszustandes in der Kriechschicht (6-8) in (18) einen Wert für $K_{\mathrm{MN}}$, erf, aus dem der erforderliche Reibungswinkel für Grenzgleichgewicht

$$
\sin \varphi_{\mathrm{erf}}=\frac{\sqrt{\left(K_{\mathrm{MN}, \text { erf }}-1\right)\left(K_{\mathrm{MN}, \text { erf }}-9\right)}}{K_{\mathrm{MN}, \text { erf }}-1}
$$

bestimmt werden kann. Für volumenkonstantes Kriechen ist $\kappa=0$. Für diesen wichtigen Fall des hangparallelen Kriechens folgt

$$
\sin \varphi_{\text {erf }}=\frac{\sqrt{18 \cos ^{2} \beta-15 \cos ^{4} \beta-3}}{5 \cos ^{2} \beta-1} .
$$

Die Auswirkung der aus den verschiedenen Grenzbedingungen folgenden erforderlichen Reibungswinkeln (16) und (20) auf die rechnerische Standsicherheit einer unendliche langen Böschung wird im nächsten Abschnitt erläutert. 


\subsection{Vergleich}

Die erforderlichen Reibungswinkel der verschiedenen Ansätze sind für böschungsparalleles Kriechen $(\kappa=0)$ in Abb. 12 dargestellt. Die Vernachlässigung der mittleren Hauptspannung im Versagenskriterium nach Mohr-Coulomb führt zum höchsten erforderlichen Reibungswinkel. Für $\beta<26,7^{\circ}$ liefert die Berechnung über das Versagenskriterium nach Matsuoka und Nakai erforderliche Reibungswinkel $\varphi_{\text {erf }}<\beta$, darüber $\varphi_{\text {erf }}>\beta$.

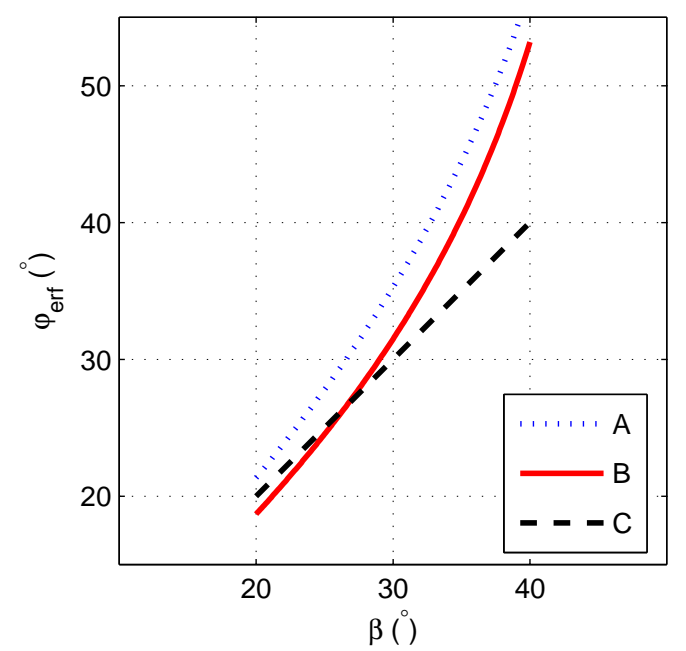

Abbildung 12: Für Grenzgleichgewicht einer kohäsionslosen unendlich langen Böschung erforderliche Reibungswinkel berechnet nach: (A) Spannungszustand nach der kinematischen Lösung für hangparalleles Kriechen und Grenzbedingung nach Mohr-Coulomb, (B) Spannungszustand nach der kinematischen Lösung für hangparalleles Kriechen und Grenzbedingung nach Matsuoka und Nakai, (C) $\varphi_{\text {erf }}=\beta$.

\subsection{Beispiel}

Für den Hochmais-Kriechhang (Abb. 1) ermittelten Schneider-Muntau und Zangerl [26] die Sicherheit mittels des Lammelenverfahrens nach Janbu [1] zu $\eta=1,1$. Die Autoren geben für das überschobenen Moränenmaterial (Kriechzone, ca. 20 dick) die Kennwerte $\gamma=24 \mathrm{kN} / \mathrm{m}^{3}, \varphi=35^{\circ}$ sowie $c=0 \mathrm{kPa}$ an. Für die darauf abgleitende Scholle aus Paragneis (im Mittel ca. 50 dick) wurden die Werte $\gamma=28 \mathrm{kN} / \mathrm{m}^{3}, \varphi=45^{\circ}$ und $c=20 \mathrm{kPa}$ verwendet.

Die Neigung der Kriechzone ist im Mittel ca. $32^{\circ}$ und in etwa böschungsparallel [24]. Die Sicherheit nach einem einfachen Modell der unendliche Böschung ist mit $\eta=\tan \left(35^{\circ}\right) / \tan \left(32^{\circ}\right)=1,12$ gut vergleichbar mit der Sicherheit nach Janbu, und die Näherung des Kriechhanges als unendliche Böschung ist vertretbar.

Für die bereits sehr lange kriechende Böschung ist es sehr wahrscheinlich, dass volumenkonstantes Kriechen vorliegt und damit ist $\kappa=0$. Damit kann der erforderliche Reibungswinkel nach (201) berechnet werden: $\varphi_{\text {erf }}=34,7^{\circ}$. Die Sicherheit $\eta=\tan \left(35^{\circ}\right) / \tan \left(34,7^{\circ}\right)=1,01$ ist zwar etwas geringer als zuvor, weist aber immer noch auf stabiles Kriechen hin. 


\section{Zusammenfassung}

Die kinematische Lösung von Haefeli [9] dient zur Ermittlung des Spannungszustandes in einer homogenen, geneigten Kriechschicht bei bekannter (gemessener) Richtung der Kriechgeschwindigkeit zur Oberfläche $\kappa$. Der somit bekannte Spannungszustand kann gemeinsam mit einem Versagenskriterium zur Abschätzung der Sicherheit einer Böschung, die als unendlich lang betrachtet werden kann, verwendet werden. Die Berechnung mit dem Versagenskriterium nach Matsuoka und Nakai liefert für volumenkonstantes Kriechen in kohäsionslosen Böden für steilere Böschung deutlich kleinere Werte für die Sicherheit als die traditionelle Lösung $\eta=\tan \varphi / \tan \beta$. Für dichte Böden wird dazu weiterhin angenommen, dass die Scherfestigkeit eines Bodens im Kriechversuch und im weggesteuertern Versuch gleich ist.

\section{Danksagung}

Ich bedanke mich bei Prof. Kolymbas für die sehr anregende und weiterführende Diskussion und bei Dr. Renk für das Erstellen der Abb. 9, Den drei anonymen Gutachter sei für die konstruktiven Anregungen und Korrekturen gedankt.

\section{Literatur}

[1] Bardet, J. (1997). Experimental Soil Mechanics. Precidence Hall, New Jersey.

[2] Brückl, E.; Zangerl, C.; Tentschert, E. (2004). Geometry and Deformation Mechanisms of a deep Seated Gravitational Creep in Crystalline Rocks. In: EUROCK 2004, Salzburg, Austria, S. 229-232.

[3] Budhu, M. (1985). Lateral Stresses Observed in Two Simple Shear Apparatus. Journal of Geotechnical Engineering, 111(6):698-711.

[4] Di Prisco, C.; Imposimato, S. (1997). Experimental analysis and theoretical interpretation of triaxial load controlled loose sand specimen collapses. Mechanics of Cohesive-frictional Materials, 2(2):93-120.

[5] Engl, D.; Fellin, W.; Zangerl, C. (2008). Scherfestigkeiten von Scherzonengesteinen Ein Beitrag zur geotechnischen Bewertung von tektonischen Störungszonen und Gleitzonen von Massenbewegungen. Bulletin für angewandte Geologie, 13(2):63-81.

[6] Feda, J. (1992). Creep of soils and related phenomena. Elsevier, Amsterdam.

[7] Germaine, G. A., T.J (2009). Geotechnical Laboratory Measurements for Engineers. John Wiley \& Sons, Inc., Hoboken, New Jersey.

[8] Goldscheider, M. (2003). Vergleichende Versuche mit einem konventionellen und einem parallel geführten Rahmenschergerät als Grundlage für DIN 18137-3. Fraunhofer IRB Verlag, Stuttgart. 
[9] Haefeli, R. (1936). Schneemechanik mit Hinweisen auf die Erdbaumechanik. Beiträge zur Geologie der Schweiz, Geotechnische Serie, Hydrologie, Lieferung 3.

[10] HAefeli, R. (1950). Investigation and measurements of the shear strengths of saturated cohesive soils. Géotechnique, 2:186-208.

[11] Janbu, N. (1954). Application of composite slip surface for stability analysis. In: Proceedings of the European Conference on Stability of Earth Slopes, S. 43-49.

[12] Kolymbas, D. (2007). Geotechnik: Grundbau und Tunnelbau. Springer, Berlin.

[13] KöRneR, H. (1969). Kinematische Betrachtungen zum Rankinschen Spannungszustand in der geneigten, kriechenden Schicht. Felsmechanik und Ingenieurgeologie, Suppl. V, S. 33-54.

[14] Krieg, S. (2000). Viskoses Bodenverhalten von Mudden, Seeton und Klei. Veröffentlichungen des Instituts für Bodenmechanik und Felsmechanik der Universität Karlsruhe, Heft 150.

[15] Leroueil, S.; Marques, M. (1996). Importance of strain rate and temperature effects in geotechnical engineering. In: Sheahan, T.; Kaliakin, V. (Hg.), Measuring and Modeling Time Dependent Soil Behavior, Nummer 61 in Geotechnical Special Technical Publication, S. 1-53. ASCE.

[16] Lindemann, M. (2003). Vergleichsversuche mit Rahmenschergeräten unterschiedlicher Bauart. geotechnik, 26(1):27-32.

[17] Matsuoka, H.; Nakai, T. (1974). Stress-deformation and strength characteristics of soil under three different principal stresses. In: Proceedings of JSCE 1974, Band 232, S. 59-74.

[18] Matsuoka, H.; Sun, D. (1995). Extension of spatially mobilized plane (SMP) to frictional and cohesive materials and its application to cemented sands. Soils and Foundation, 35(4):63-72.

[19] Mitchell, J. (1976). Fundamentals of soil behavior. Series in soil engineering. Wiley, New York.

[20] Oberhollenzer, D. (2009). Einfluss der Seitenspannung auf das Kriechverhalten von Böden im Triaxialversuch. Diplomarbeit, Universität Innsbruck.

[21] Renk, D. (2006). Geotechnische Untersuchungen von Gleitzonenmaterialien großer Hangbewegungen. Diplomarbeit, Universtität Karlsruhe.

[22] Rowe, P. W. (1969). The Relation Between the Shear Strength of Sands in Triaxial Compression, Plane Strain and Direct Shear. Géotechnique, 19(1):75-86.

[23] Schneider-Muntau, B. (in Arbeit). Numerische Modellierung von Kriechhängen. Dissertation, Universität Innsbruck. 
[24] Schneider-Muntau, B.; Katzenbach, R.; Fellin, W. (2005). Numerical Modelling of a Creeping Landslides: Applicability of Material Properties determined in Laboratory Experiments. In: International Symposium on Latest Natural Disasters - New Challenges for Engineering Geology, Geotechnics and Civil Protection, Sofia, Bulgaria.

[25] Schneider-Muntau, B.; Renk, D.; Marcher, T.; Fellin, W. (2006). The Importance of Laboratory Experiments for Finite Element Calculations in Landslide Investigations. In: Geohazards - Technical, Economical and Social Risk Evaluation, 18. - 21. Juni, Lillehammer, Norwegen.

[26] Schneider-Muntau, B.; Zangerl, C. (2005). Numerical Modelling of a Slowly Creeping Landslide in Crystalline Rock - a Case Study. In: ISRM Regional Symposium Eurock 2005, Brno, Czech Republic, S. 535-540.

[27] Shibuya, S.; Mitachi, T.; Tamate, S. (1997). Interpretation of direct shear box testing of sands as quasi-simple shear. Géotechnique, 47:769-790.

[28] Singh, A.; Mitchell, J. (1986). General stress-strain-time function for soils. ASCE Journal of Soil Mechanics \& Foundations Div, 94(1):21-46.

[29] TaYlor, D. (1948). Fundamentals of Soil Mechanics. John Wiley \& Sons, Inc., New Jork, London.

[30] Thornton, C.; Zhang, L. (2003). Numerical Simulations of the direct shear test. Chemical Engineering \& Technology, 26(2):153-156.

[31] Thornton, C.; Zhang, L. (2006). A numerical examination of shear banding and simple shear non-coaxial flow rules. Philosophical Magazine, 86:3425-3452.

[32] Vulliet, L.; Hutter, K. (1988). Set of constitutive models for soils under slow movement. Journal of Geotechnical Engineering, 114(9):1022-1041.

[33] Vulliet, L.; Hutter, K. (1988). Viscous-type sliding laws for landslides. Canadian Geotechnical Journal, 25(3):467-477.

\section{A. Kinematische Lösung nach Haefeli}

Haefeli 9] entwickelte eine kinematische Lösung zur Berechnung des Spannungszustandes in einer geneigten Schneeschicht. Er hat seine Lösung graphisch gefunden und lediglich Aussagen über die Spannungen in einer Schnittebene längs des Hanges getroffen. Hier wird eine algebraisch abgeleitete auf den vollständigen Spannungstensor erweiterte Lösung präsentiert.

Haefeli [9] hat aus Messungen in Schneedecken gefunden,

- dass alle Kriechgeschwindigkeiten annähernd parallel und um den Kriechwinkel $\kappa$ zur Schneedecke geneigt sind; 
- in einer sehr langen Böschung mit konstanter Neigung alle Kriechprofile kongruent sind, d.h die Kriechgeschwindigkeiten hängen nicht von $x_{1}$ (Abb. 13) ab.

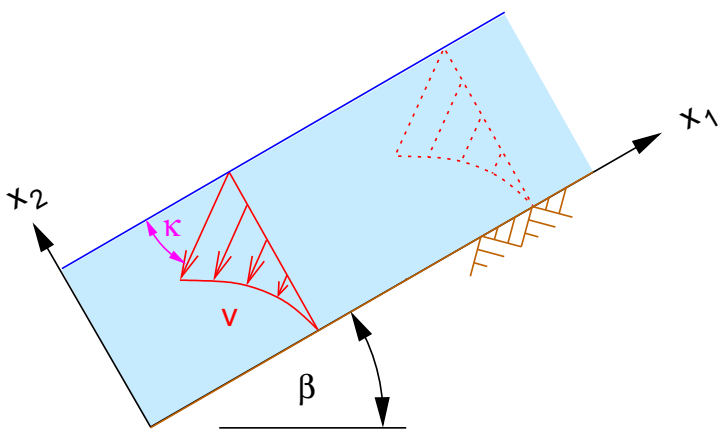

Abbildung 13: Voraussetzungen für die kinematische Lösung.

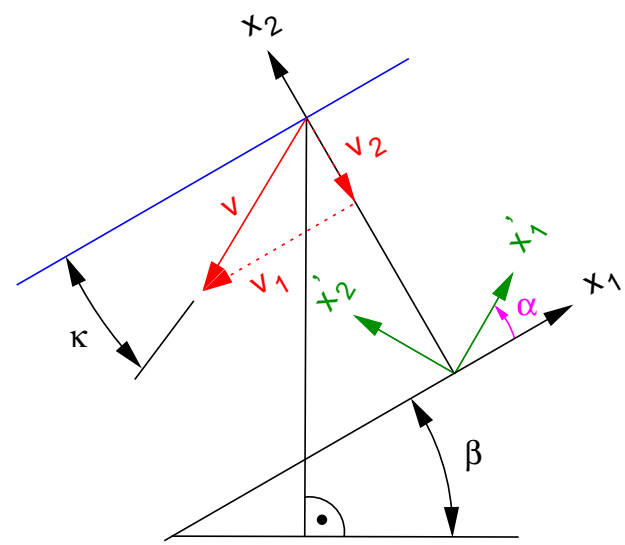

Abbildung 14: Winkeldefinitionen.

Haefeli hat nun für einen aus Messungen bekannten Kriechwinkel $\kappa$ den Spannungszustand in der kriechenden Schicht (Schneedecke) vollständig bestimmt. Er hat dazu auch die Linearität der Kriechprofile vorausgesetzt, diese ist aber für die folgende Berechnung nicht notwendig. Die Kriechgeschwindigkeit kann einen beliebigen Verlauf über die Tiefe haben: $\mathbf{v}\left(x_{2}\right)$.

Die Hauptrichtungen der Spannung und der Verzerrungsrate: Für ein viskoses 3 Material gilt, dass Schubspannungen Schubverzerrungsraten hervorrufen. Hauptspannungsrichtungen sind schubspannungsfrei und für ein viskoses Material folglich auch schubverzerrungsratenfrei. Die Hauptrichtungen des Spannungstensors und des Verzerrungsratentensors sind also gleich, d.h. $\boldsymbol{\sigma}$ und $\mathbf{D}$ sind koaxial.

Das Geschwindigkeitsfeld 5 ist (Abb. 14):

$$
\mathbf{v}=\left(\begin{array}{c}
v_{1} \\
v_{2}
\end{array}\right)=\mathbf{v}_{0} f\left(x_{2}\right)=\left(\begin{array}{c}
\cos \kappa \\
\sin \kappa
\end{array}\right) f\left(x_{2}\right)
$$

Darin ist $f$ eine beliebige skalare Funktion von $x_{2}$, welche die Größe der Geschwindigkeit in jeder Tiefe angibt. Die Richtung der Geschwindigkeitsvektoren ist durch den Winkel $\kappa$ gegeben und wird als Vektor $\mathbf{v}_{0}$, mit $\left|\mathbf{v}_{0}\right|=1$, eingeführt.

Der Verzerrungsratentensor $\mathbf{D}$ ist allgemein

$$
D_{i j}=\frac{1}{2}\left(\frac{\partial v_{j}}{\partial x_{i}}+\frac{\partial v_{i}}{\partial x_{j}}\right) .
$$

\footnotetext{
${ }^{3}$ Für diese Ableitung ist eine Einschränkung auf lineare Viskosität nicht notwendig.

${ }^{4}$ Der Verzerrungsratentensors ist der symmetrische Anteil des Geschwindigkeitsgradienten.

${ }^{5}$ Da Kompression als positiv definiert ist, sind Geschwindigkeiten entgegen der Koordinatenrichtung positiv.
} 
Durch Gradientenbildung folgt aus (21):

$$
\mathbf{D}=\frac{\partial f\left(x_{2}\right)}{\partial x_{2}}\left(\begin{array}{cc}
0 & \frac{1}{2} \cos \kappa \\
\frac{1}{2} \cos \kappa & \sin \kappa
\end{array}\right)
$$

Die Eigenvektoren dieses Tensors sind die gesuchten Hauptrichtungen. Dazu wird der Verzerrungsratentensor mit

$$
\mathbf{Q}=\left(\begin{array}{cc}
\cos \alpha & \sin \alpha \\
-\sin \alpha & \cos \alpha
\end{array}\right)
$$

in ein gedrehtes Koordinatensystem $\mathbf{x}^{\prime}$ transformiert $\left(x_{1}^{\prime}, x_{2}^{\prime}\right.$ in Abb. 14)

$$
\mathbf{D}^{\prime}=\mathbf{Q D Q}^{\mathrm{T}}
$$

Die Koordinatenrichtungen $x_{1}^{\prime}$ und $x_{2}^{\prime}$ sind die Hauptrichtungen, wenn $D_{12}^{\prime}=0$ gilt. Diese Bedingung ergibt

$$
\alpha=\frac{\pi}{4}+\frac{\kappa}{2} .
$$

Für diesen Winkel gilt auch $D_{11}^{\prime} \geq D_{22}^{\prime}$.

Die Hauptspannungen: Nun sind die Normalspannung und die Schubspannung in einer Eben normal zu $x_{2}$ (3) sowie die Hauptrichtungen (26) bekannt. Aus

$$
\left(\begin{array}{cc}
\sigma_{11}^{\prime} & 0 \\
0 & \sigma_{22}^{\prime}
\end{array}\right)=\boldsymbol{\sigma}^{\prime}=\mathbf{Q} \boldsymbol{\sigma} \mathbf{Q}^{\mathrm{T}}=\mathbf{Q}\left(\begin{array}{cc}
\sigma_{11} & p_{v} \sin \beta \\
p_{v} \sin \beta & p_{v} \cos \beta
\end{array}\right) \mathbf{Q}^{\mathrm{T}}
$$

folgen mit $\sigma_{12}^{\prime}=0$ die noch unbekannte Spannung

$$
\sigma_{11}=\frac{2 \sigma_{12} \cos ^{2} \alpha+\sigma_{22} \cos \alpha \sin \alpha-\sigma_{12}}{\cos \alpha \sin \alpha}=p_{v} \frac{\cos (\beta+\kappa)-\sin (\beta) \sin (\kappa)}{\cos \kappa}
$$

und damit die Hauptspannungen

$$
\begin{aligned}
\sigma_{I} & :=\sigma_{11}^{\prime}=\frac{\sigma_{12} \cos \alpha+\sigma_{22} \sin \alpha}{\sin \alpha} \\
\sigma_{I I I} & :=\sigma_{22}^{\prime}=\frac{-\sigma_{12} \sin \alpha+\sigma_{22} \cos \alpha}{\cos \alpha} .
\end{aligned}
$$

Einsetzen von (3), (44) und (26) ergibt

$$
\begin{aligned}
\sigma_{I} & =p_{v} \frac{\cos (\beta+\kappa)+\sin (\beta)}{\cos \kappa} \\
\sigma_{I I I} & =p_{v} \frac{\cos (\beta+\kappa)-\sin (\beta)}{\cos \kappa} .
\end{aligned}
$$

Werden die Hauptspannungen direkt berechnet, kann $\sigma_{11}$ aus der Rücktransformation

$$
\boldsymbol{\sigma}=\mathbf{Q}^{\mathrm{T}} \boldsymbol{\sigma}^{\prime} \mathbf{Q}
$$


berechnet werden

$$
\sigma_{11}=\sigma_{I} \cos ^{2} \alpha+\sigma_{I I I} \sin ^{2} \alpha
$$

Der volle Spannungstensor: Der volle Spannungstensor lässt sich aus Überlegungen zu den Randbedingungen finden. Wegen des ebenen Verformungszustandes verschwinden die Verzerrungsraten quer zum Hang: $D_{33}=0, D_{13}=D_{23}=0$. Damit gilt $\sigma_{13}=\sigma_{23}=0$. In Richtung des Hanges $\left(x_{1}\right)$ ist wegen der unendlichen Ausdehnung $D_{11}=0$. Die Normalverzerrung ist also sowohl quer zum Hang als auch in Richtung des Hanges behindert. Daraus folgt für ein isotropes Material $\sigma_{11}=\sigma_{33}$. Diese Spannung ist die noch fehlende Hauptspannung $\sigma_{I I}:=\sigma_{11}$, da $\sigma_{13}=\sigma_{23}=0$, und liegt wegen (34) zwischen den zuvor ermittelten Hauptspannungen $\sigma_{I}$ und $\sigma_{I I I}$ :

$$
\sigma_{I I}=p_{v} \frac{\cos (\beta+\kappa)-\sin (\beta) \sin (\kappa)}{\cos \kappa} .
$$

Hauptspannungen für hangparalleles Kriechen: Für den wichtigen Spezialfall $\kappa=0$ ergeben sich die Hauptspannungen zu

$$
\begin{aligned}
\sigma_{I} & =p_{v}(\cos \beta+\sin \beta) \\
\sigma_{I I} & =p_{v} \cos \beta \\
\sigma_{I I I} & =p_{v}(\cos \beta-\sin \beta) .
\end{aligned}
$$

Berücksichtigung einer Grundwasserströmung: Für eine hangparallele Grundwasserströmung folgen aus dem effektiven Lammellengewicht $G^{\prime}=\gamma^{\prime} t b \cos \beta$ und der Strömungskraft $F_{s}=\left(\gamma_{w} \sin \beta\right) t b \cos \beta$ die effektiven Spannungen

$$
\begin{aligned}
\sigma_{22} & =\frac{G^{\prime} \cos \beta}{b}=\gamma^{\prime} t \cos ^{2} \beta \\
\sigma_{21} & =\frac{G^{\prime} \sin \beta+F_{s}}{b}=\left(\gamma^{\prime}+\gamma_{w}\right) t \cos \beta \sin \beta=\gamma_{r} t \cos \beta \sin \beta .
\end{aligned}
$$

Diese effektiven Spannungen können in (29) und (30) eingesetzt werden, um die Hauptspannungen zu erhalten. Der Winkel $\alpha$ ist durch das Geschwindigkeitsfeld gegeben und ändert sich deshalb nicht.

\section{B. Zur Bestimmung der Scherfestigkeit}

Zur Bestimmung der Scherfestigkeitsparameter, Kohäsion und Reibungswinkel, bieten sich Triaxial-, Rahmenscher- und in seltenen Fällen Einfachscherversche an, Abb. 15, Wir untersuchen abschließend die Vergleichbarkeit der Ergebnisse dieser Versuche für kohäsionslose Böden. Dazu beschränken wir uns auf wesentliche Unterschiede in der Interpretation der Ergebnisse. Weitere Schwierigkeiten entstehen dadurch, dass die Ergebnisse von Rahmenscherversuchen stark von der Bauweise des Gerätes abhängen [8, 16].

Auswertung: Im Rahmenscher- bzw. Einfachscherversuch werden aus Normal- und Schubkraft die gemittelte Normalspannung $\sigma$ und die gemittelte Schubspannung $\tau$ berechnet. Das bei der Scherung 

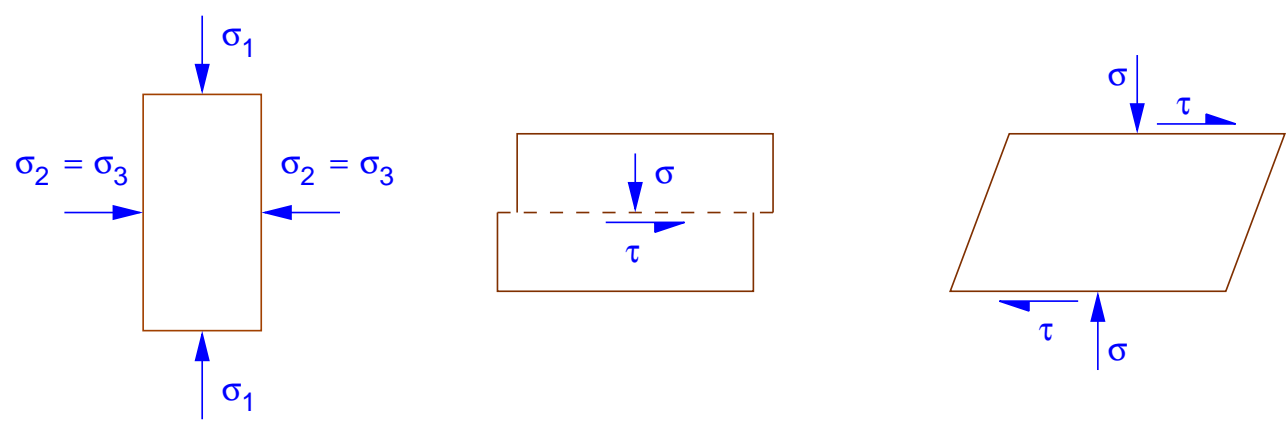

Abbildung 15: Triaxial-, Rahmenscher- und Einfachscherversuch schematisch.

erreichte maximale Verhältnis $(\tau / \sigma)_{\max }$ entspricht einem Reibungswinkel $\phi$

$$
\tan \phi:=\left(\frac{\tau}{\sigma}\right)_{\max }
$$

Üblicherweise wird ein Hauptspanungszustand wie in Abb. 16(a) angenommen [z.B. 7, 29].

Auf Hill zurückgeführt wird die Annahme, dass sich die Scherfuge des Rahmenscherversuches wie eine Einfachscherung ausbildet, in welcher ein um $45^{\circ}$ gedrehter Hauptspannungzustand $\sigma_{I}=\sigma+\tau$ und $\sigma_{I I I}=\sigma-\tau$ vorliegt, siehe Abb. 16(b). Für Scherung im kritischen Zustand, d.h. volumenkonstantes Scheren, wurde die Drehung der gemittelten Hauptspannungen im Rahmenscherversuch um $45^{\circ}$ von Thornton und Zhang [30] mittels der Diskreten-Elemente-Methode nachgewiesen. Setzen wir diese Hauptspannungen in die Grenzbedingung nach Mohr-Coulomb (15) für $c=0$ ein, so folgt

$$
\sin \varphi=\left(\frac{\sigma_{I}-\sigma_{I I I}}{\sigma_{I}+\sigma_{I I I}}\right)_{\max }=\left(\frac{\tau}{\sigma}\right)_{\max }
$$

Aus dem Vergleich von (42) mit (41) erkennen wir

$$
\sin \varphi=\tan \phi \leadsto \varphi \geq \phi
$$

Für Peakzustände haben Thornton und Zhang [31] Hauptspannungen zwischen den Extremen in Abb. 16(b) und 16(a) berechnet. Dies bedeutet, dass der üblicherweise angegeben Reibungswinkel $\phi$ praktisch immer kleiner ist als der aus der Grenzbedingung nach Mohr-Coulomb folgende Reibungswinkel $\varphi$. Dies hat bereits Rowe [22] erkannt und gibt an

$$
\tan \phi=\tan \varphi \cos \varphi_{c}
$$

Die Dilatanz eines dichten Bodens wird hier über den Unterschied von $\varphi$ zum kritischen Reibungswinkel $\varphi_{c}$ erfasst. Für volumenkonstante Scherung ist $\varphi=\varphi_{c}$ und es folgt direkt (43).

Da im Rahmenscherversuch ein ebener Verformungszustand herrscht folgt, dass die Auswertung mit (41) den Reibungswinkel für ebene Verformung theoretisch unterschätzt. Dies kann sich allerdings durch bauartbedingte Zwängungen (z.B. geführte Rahmen) eventuell auch umdrehen.

Eine weitere Fehlerquelle ist ein zu kleiner Scherspalt im Rahmenschergerät. Der Spalt sollte nach Shibuya et al. [27] eine Höhe von $(10 \ldots 20) \cdot d_{50}$ aufweisen, mit $d_{50}$ dem Korndurchmesser bei $50 \%$ 


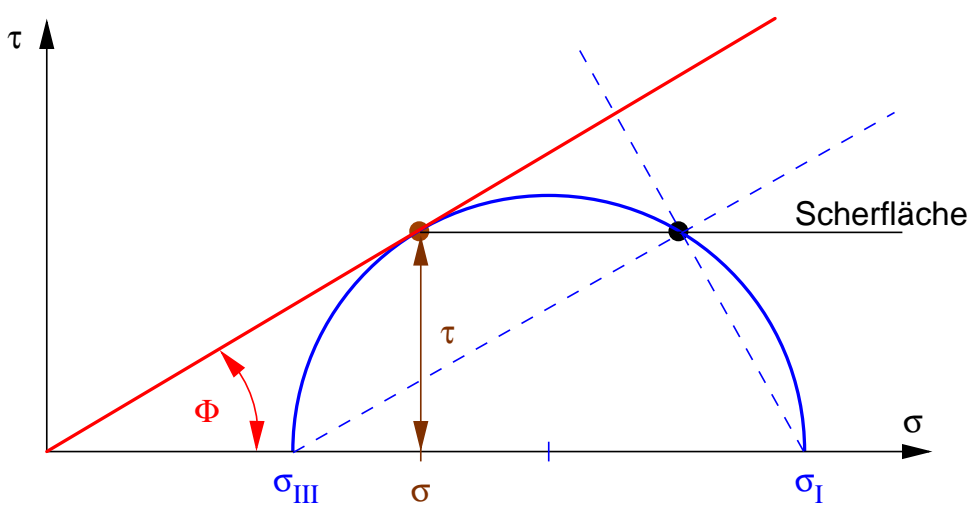

(a) Übliche Interpretation.

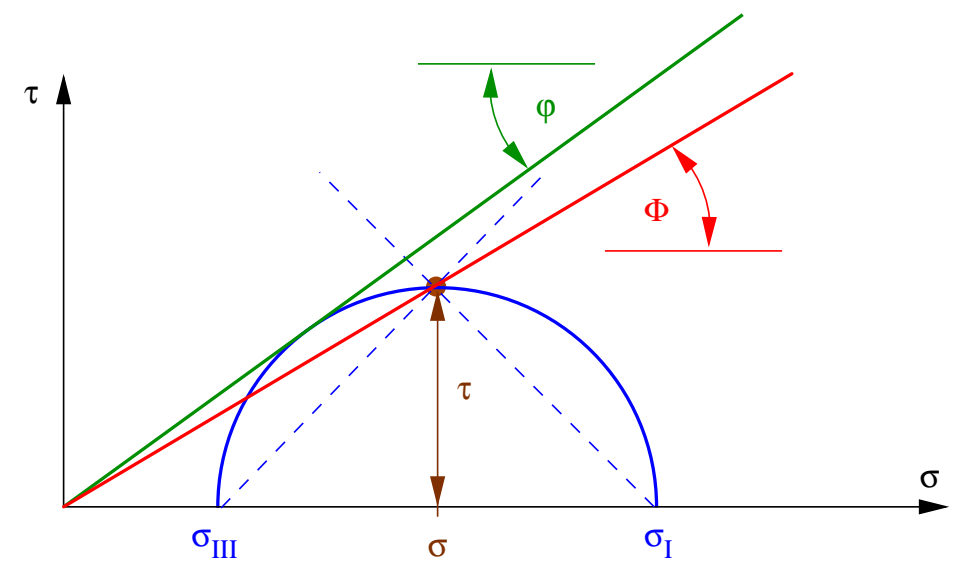

(b) Auf Hill zurückgeführte Interpretation.

Abbildung 16: Interpretationen der Auswertung eines Rahmenscherversuches: Hauptspannungen und Ebenen der Hauptspannungen. 
Siebdurchgang. Für geringere Spalthöhen nimmt der bei Rahmenscherversuchen gemessene PeakReibungswinkel laut Shibuva et al. zu. Damit sind eigentlich alle konventionellen kleineren Schergeräte nur für sehr feinkörnige Böden geeignet.

Vergleich zwischen Triaxial- und Rahmenscherversuch: Im triaxialen Kompressionsversuch werden die über den Querschnitt gemittelten Vertikalspannung $\sigma_{1}$ und die Horizontalspannungen $\sigma_{2}=\sigma_{3}$ bestimmt, welche den größten und kleinsten Hauptspannungen $\sigma_{I}=\sigma_{1}$ und $\sigma_{I I I}=\sigma_{3}$ entsprechen. Über das Mohr-Coulomb'sche Bruchkriterium (15) kann ein Reibungswinkel $\varphi$ errechnet werden

$$
\sin \varphi_{\mathrm{TR}}:=\left(\frac{\sigma_{1}-\sigma_{3}}{\sigma_{1}+\sigma_{3}}\right)_{\max } .
$$

Für einen Vergleich zwischen Triaxial- und Rahmenscherversuch muss berücksichtigt werden, dass im Rahmenscherversuch ein ebener Verformungszustand herrscht. Für diesen liefert das Versagenskriterium nach Mohr-Coulomb-Kriterium höhere Reibungswinkel als für triaxiale Bedingungen (vgl. Abb. 111).

Rowe [22] gibt den Zusammenhang an

$$
\tan \phi=\tan \left(1,12 \varphi_{\mathrm{TR}}\right) \cos \varphi_{c}
$$

Damit kann der Reibungswinkel im Rahmenscherversuch $\phi$ größer oder kleiner als der Reibungswinkel $\varphi_{\mathrm{TR}}$ im Triaxialversuch werden.

Einfachscherversuch: Ähnliches ergibt sich auch für Einfachscherversuche. Eine exemplarische Nachrechnung eines Versuches von Budhu [3] mit dichtem Sand ergibt einen um ca. $38^{\circ}$ gedrehten Hauptspannungszustand am Peak und folgende Peakwerte: $\varphi=55,4^{\circ}$ und $\phi=43,1^{\circ}$. Im Einfachschergerät ist wegen der behinderten Querdehnung $\sigma_{I I}>\sigma_{I I I}$. Deshalb muss mit dem Versagenskriterium nach Matsuoka-Nakai ausgewertet werden. Nach Berechnung der Hauptspannungen aus den gemessenen Vertikal-, Horizontal- und Schubspannungen folgt durch Einsetzen in (18) ein Wert für $K_{\mathrm{MN}}$ und weiter

$$
\varphi_{\mathrm{MN}}=\arcsin \left(\frac{\sqrt{\left(K_{\mathrm{MN}}-1\right)\left(K_{\mathrm{MN}}-9\right)}}{K_{\mathrm{MN}}-1}\right),
$$

vgl. (19). Dies ergibt für die Versuche $\varphi_{\mathrm{MN}}=50,5^{\circ}$.

Bezug zum Spannungszustand in einer Kriechschicht: Die kinematische Lösung von Haefeli liefert für volumenkonstantes Kriechen $(\kappa=0)$ einen um $45^{\circ}$ zur Scherrichtung gedrehten Hauptspannungszustand, vgl. (26) . Dies stimmt gut mit den Richtungen der Hauptspannungen bei volumenkonstanter Scherung im kritischen Zustand überein. Für stark dilatantes Kriechen, $\kappa=-\varphi$, ergeben sich Hauptspannungen wie in Abb. 16(a).

Auswirkung auf Standsicherheitsberechnungen von Böschungen: Da der im Rahmenscherversuch ermittelte Reibungswinkel $\phi$ eher unsicher zu interpretieren ist, sollten eventuell größere Sicherheiten in einer Stabilitätsanalyse angewendet werden. 